\title{
Alkane Coiling in Perfluoroalkane Solutions: A New Primitive Solvophobic Effect
}

Pedro Morgado, ${ }^{\dagger}$ Ana Rosa Garcia, ${ }^{\ddagger, \S}$ Luís F. G. Martins, ${ }^{\dagger, \|}$ Laura M. Ilharco, ${ }^{*, \oplus}$ and Eduardo J. M. Filipe ${ }^{*} \oplus$

${ }^{\dagger}$ Centro de Química Estrutural, Instituto Superior Técnico and ${ }^{\ddagger}$ Centro de Química-Física Molecular and Institute of Nanoscience and Nanotechnology, Instituto Superior Técnico, Universidade de Lisboa, 1049-001 Lisboa, Portugal

${ }^{\S}$ Departamento de Química e Farmácia, FCT, Universidade do Algarve, 8000 Faro, Portugal

"Centro de Química de Évora, Escola de Ciências e Tecnologia, Universidade de Évora, 7000-671 Évora, Portugal

\section{Supporting Information}

ABSTRACT: In this work, we demonstrate that $n$-alkanes coil when mixed with perfluoroalkanes, changing their conformational equilibria to more globular states, with a higher number of gauche conformations. The new coiling effect is here observed in fluids governed exclusively by dispersion interactions, contrary to other examples in which hydrogen bonding and polarity play important roles. FTIR spectra of liquid mixtures of $n$-hexane and perfluorohexane unambiguously reveal that the population of $n$-hexane molecules in all-trans conformation reduces from $32 \%$ in the pure $n$-alkane to practically zero. The spectra of perfluorohexane remain unchanged, suggesting nanosegregation of the hydrogenated and fluorinated chains. Molecular dynamics simulations support this analysis. The new solvophobic effect is prone to have a major impact on the structure, organization, and therefore thermodynamic properties and phase equilibria of fluids involving mixed hydrogenated and fluorinated chains.

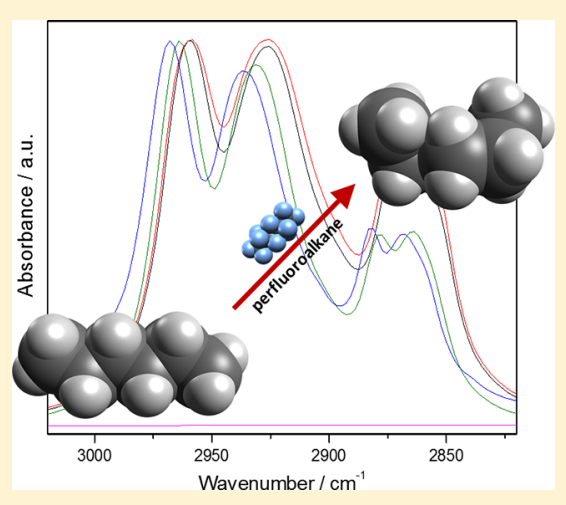

\section{INTRODUCTION}

The peculiar properties of mixtures of alkanes and perfluoroalkanes have been intriguing the liquids/fluids community since the pioneering work of Scott. ${ }^{1}$ Given the similarity of the two components in terms of molecular structure and intermolecular forces, mixtures of alkanes and perfluoroalkanes could be expected to be the ultimate example of an ideal mixture. On the contrary, they show an enhanced tendency to phase separate, exhibiting extensive regions of liquid-liquid immiscibility, large positive deviations from Raoult's law, and large positive excess enthalpy and volume.

The changes in volume that occur when hydrogenated and fluorinated substances are mixed are impressive: the excess volume of the equimolar mixture of $n$-hexane and perfluorohexane is $5 \mathrm{~cm}^{3} \mathrm{~mol}^{-1}$; when perfluorohexane is immersed in $n$-octane at infinite dilution, its molar volume increases by $13 \%{ }^{2,3}$ The effect is even more pronounced when $n$-alkanes are dissolved in perfluoroalkanes: their molar volumes increase by $20 \% !^{4}$ Literally, thin layers of empty space are created around each molecule.

This unusual behavior extends to transport and surface properties. We have recently shown that the viscosity of (hexane + perfluorohexane) mixtures is $17 \%$ lower than expected. ${ }^{5}$ As for interfacial behavior, ( $n$-hexane + perfluorohexane) mixtures display a minimum on the surface tension vs composition curve, a rare phenomenon called aneotropy. ${ }^{6,7}$
The atypical properties of mixtures of alkanes and perfluoroalkanes are far from being a mere scientific curiosity. The thermophysical and mechanical properties of fluorinated compounds, in particular fluorinated surfactants, indicate that they may be used in numerous applications in chemical engineering and material sciences. Thus, the presence of fluorinated materials in everyday life is pervasive. However, owing to their inertness and biocompatibility, it is in biomedical $\mathrm{R} \& \mathrm{D}$ that fluorinated substances find their most exciting applications as components in blood substitute formulations, emulsions for drug delivery in liquid ventilation, and components of microbubbles for drug delivery and oxygen transport. $^{8-11}$

Additionally, the simultaneous presence of the mutually phobic hydrogenated and perfluorinated segments can dramatically influence the structure of the fluid/material, inducing new forms of organization. Semifluorinated alkanes, for example, which are diblock compounds of covalently bonded alkyl and perfluoroalkyl segments, display amphiphilic character, forming different types of supramolecular structures. $^{12-17}$ Given the lack of polarity or groups able to make

Special Issue: Tribute to Keith Gubbins, Pioneer in the Theory of Liquids

Received: July 19, 2017

Revised: September 12, 2017

Published: September 18, 2017 
hydrogen bonds, these compounds have been often called primitive surfactants. It should be emphasized that, in this case, amphiphilicity is the result of a subtle balance between weak dispersion forces. We have recently accomplished a systematic study of the thermophysical properties of liquid semifluorinated alkanes, either pure or in mixtures, which are crucial to characterizing and modeling systems with fluorinated chains in general. $^{18-21}$

While a definitive explanation for this intriguing behavior is still awaited, it is generally interpreted as an indication of weaker than expected dispersion interactions between hydrocarbons and fluorocarbons. ${ }^{22-24}$ Most of the evidence comes from theoretical modeling, in which matching the experimental results implies the use of parameters that lower the intermolecular dispersion energy, ${ }^{25-27}$ Structural factors, such as the rigidity of fluorinated chains, as opposed to the characteristic flexibility of hydrogenated chains, may also contribute to the observed behavior. ${ }^{28}$ Additionally, it has been found that, in molecular dynamics simulations, a reduction of $\sim 10 \%$ of the total dispersive interactions has almost no effect on the simulated excess volumes. ${ }^{24,29,30}$ This uncovered that, contrary to what is usually assumed, the volume increase in these mixtures is not entirely related to weak dispersive interactions and should be associated with the repulsive part of the intermolecular potential.

One of the main differences between hydrogenated and fluorinated chains is conformational. $n$-Alkanes and perfluoroalkanes may exist in different conformers, whose diversity increases with chain length. In the case of $n$-hexane, these range from all-trans to three bonds with gauche rotations. In the solid state, $n$-hexane is entirely all-trans, while in the liquid state the populations of other conformers are significant. ${ }^{31,32}$ It has been estimated that the conformer with a terminal gauche rotation (TTG) corresponds to $\sim 35 \%$, the all-trans corresponds to $\sim 32 \%$, and the TGT corresponds to $\sim 18 \% .^{33,34}$ At room temperature, conformers with two and three gauche rotations are estimated to be less than $15 \%$ and less than $1 \%$, respectively. On their turn, $n$-perfluoroalkanes are known to exist in a helical conformation with $\mathrm{C}-\mathrm{C}-\mathrm{C}-\mathrm{C}$ dihedral angles of $\sim 13^{\circ} .{ }^{35-39}$ In the solid phase, the more abundant population of conformers of $n$-perfluoroalkanes is in the spiral conformation. $^{35,36}$ Thus, a real TTT conformer is not predicted for these molecules. ${ }^{37}$ This twisted spatial arrangement was initially attributed to repulsive van der Waals and electrostatic interactions between fluorine atoms, but more recent studies point to hyperconjugation as the cause of helicity. ${ }^{38,39}$

Valuable information on the conformational equilibria, both in pure components and binary mixtures, may be retrieved from vibrational spectra, as changes in chain conformations induced by a second component may be assessed by spectral analysis. For example, for $n$-hexane, the vibrational modes observable in the infrared spectrum depend on the conformational equilibrium. For the most abundant conformer, TTG $\left(\mathrm{C}_{1}\right.$ point group), and for TGT ( $C_{2}$ point group), all 54 fundamental modes are infrared-active, whereas for the TTT conformer $\left(C_{2 h}\right.$ point group) only 27 normal modes are observable. ${ }^{40}$ The spectral regions corresponding to $\nu \mathrm{CH}, \nu \mathrm{CC}$, and/or $\delta \mathrm{CH}$ modes have been widely used to obtain direct information about the conformations of $n$-hexane. ${ }^{41-44}$

In a recent paper, we observed the coiling of alkyl chains in mixtures of hydrogenated and fluorinated alcohols. ${ }^{45}$ However, in the case of alcohols, coiling could also be due to the presence of the strong hydrogen bonds and polar interactions. The present work was designed to eliminate that possibility. We found that mixing with fluorocarbons induces conformational changes in $n$-alkanes, from all-trans to more globular arrangements involving gauche conformations. This result was obtained from the quantitative analysis of the infrared spectra of ( $n$ hexane + perfluorohexane) mixtures, and to the best of our knowledge, it is the first time that such a coiling effect has been observed and reported in fluids governed exclusively by dispersion interactions. In terms of liquid structure, the interpretation of the results is further supported by molecular dynamics simulations.

\section{EXPERIMENTAL SECTION}

All of the compounds used, $n$-hexane, $n$-octane, and perfluorohexane, were supplied by Sigma-Aldrich with stated purities better than $99 \%$, and no further purification was attempted. The $n$-hexane + perfluorohexane mixtures were prepared by weight in screw-cap vials. For the $n$-octane + perfluorohexane system, appropriate amounts of both components were mixed in a screw-cap vial in order to obtain approximately equal volumes of the two immiscible phases. The vial was then stirred and left to decant at room temperature, and samples of the coexisting phases were collected with a hypodermic syringe.

The IR spectra of the pure components and binary mixtures were obtained in transmission mode, using a Mattson Research Series 1 FTIR spectrometer with a wide-band MCT detector (400-4000 $\mathrm{cm}^{-1}$ ), with resolution $2 \mathrm{~cm}^{-1}$. The samples were analyzed by placing 2 drops between polished $\mathrm{KBr}$ disks. Each spectrum is the result of ratioing 50 coadded scans for the sample to the same number of background scans (clean $\mathrm{KBr}$ disks). The spectra are presented in absorbance units without baseline correction.

\section{RESULTS AND DISCUSSION}

The infrared spectra of pure $n$-hexane and perfluorohexane (PFH) are shown in Figure 1. In the 3100-2700 and 1500-

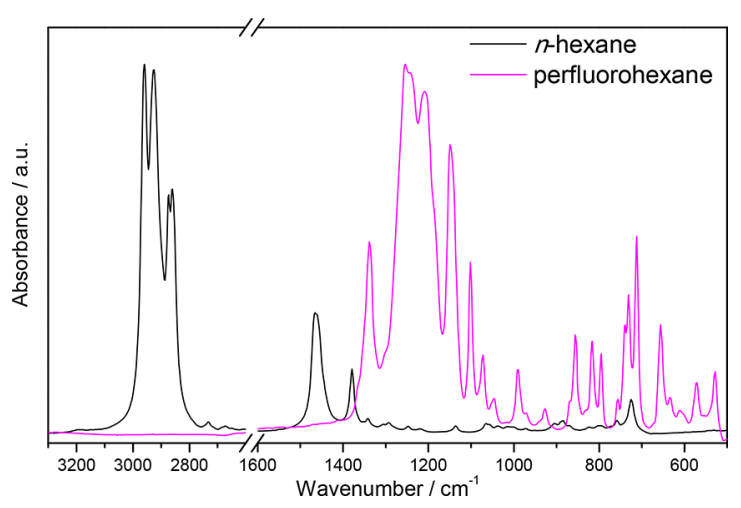

Figure 1. Transmission infrared spectra of $n$-hexane and perfluorohexane, normalized to the maximum absorption.

$1400 \mathrm{~cm}^{-1}$ spectral regions, only $n$-hexane absorbs; the $1400-$ $1150 \mathrm{~cm}^{-1}$ region corresponds almost exclusively to $\mathrm{PFH}$ bands, and in the $1000-500 \mathrm{~cm}^{-1}$ region, the two components' bands overlap. The complete band assignment is summarized in Table S1 of the Supporting Information. ${ }^{35,46-48}$

For a detailed analysis, these three spectral regions are presented separately for the pure components and binary mixtures in Figure 2. In the low-frequency region (Figure 2C), the bands assigned to $\mathrm{PFH}$ are stronger and more numerous. The spectra of mixtures richer in one component, $x$ ( $n$-hexane) $=0.8$ and $x(\mathrm{PFH})=0.8$, are very similar to those of pure $n$ hexane and $\mathrm{PFH}$, respectively. The main changes caused by 

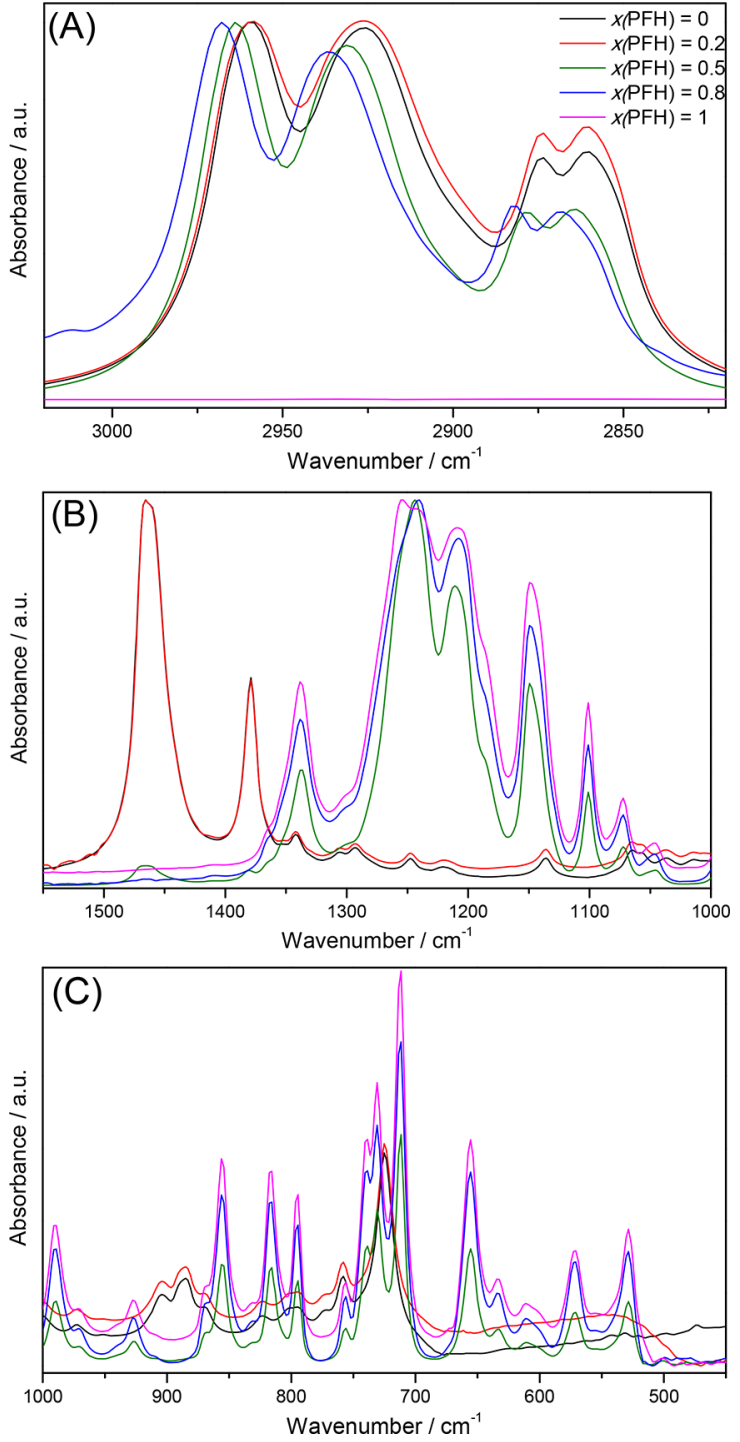

Figure 2. Three regions of the IR spectra of $n$-hexane, perfluorohexane $(\mathrm{PFH})$, and their binary mixtures, normalized to the maximum absorption in the (A) $3100-2700 \mathrm{~cm}^{-1}$ region and (B and C) $1500-$ $1200 \mathrm{~cm}^{-1}$ region, with (C) multiplied by 2 .

mixing are observed in the $\mathrm{C}-\mathrm{H}($ Figure $2 \mathrm{~A}$ ) and $\mathrm{C}-\mathrm{F}$ (Figure 2B) stretching regions.

The four bands of pure $n$-hexane in Figure $2 \mathrm{~A}$ are assigned to stretching modes of methyl and methylene groups: antisym- metric $\nu_{\mathrm{as}} \mathrm{CH}_{3}\left(2959 \mathrm{~cm}^{-1}\right)$ and $\nu_{\mathrm{as}} \mathrm{CH}_{2}\left(2926 \mathrm{~cm}^{-1}\right)$ and symmetric $\nu_{\mathrm{s}} \mathrm{CH}_{3}\left(2874 \mathrm{~cm}^{-1}\right)$ and $\nu_{\mathrm{s}} \mathrm{CH}_{2}\left(2861 \mathrm{~cm}^{-1}\right)$. These bands are sensitive to conformations, ${ }^{42}$ but not in a quantitative way, due to the possibility of combination bands and Fermi resonance with overtones of deformation modes $\left(2 \times \delta_{\text {sc }} \mathrm{CH}_{2}\right.$ or $\left.2 \times \delta \mathrm{CH}_{3}\right)$. Nevertheless, it is commonly accepted that a shift of these bands to higher wavenumbers is associated with an increased population of conformers with gauche rotations. ${ }^{41,42}$ The changes observed in Figure $2 \mathrm{~A}$ with increasing $\mathrm{PFH}$ molar ratio suggest the alteration of the conformation equilibrium toward gauche conformers.

The methyl and methylene deformations (Figure 2B) can also contribute to the analysis of $n$-hexane conformers, in particular, the relative intensity of the methylene scissors mode of the TTT conformer. ${ }^{34,44}$ Since the $\delta_{\text {as }} \mathrm{CH}_{3}$ and $\delta_{\text {sc }} \mathrm{CH}_{2}$ bands overlap, the spectral analysis in this region is not straightforward.

In an attempt to quantify the effects of mixing with PFH on the spectrum of $n$-hexane, a band deconvolution was performed in two spectral regions $\left(3020-2820\right.$ and $1510-1420 \mathrm{~cm}^{-1}$ ) by a nonlinear least-squares fitting method using a sum of Voigt components. For pure $n$-hexane, it was possible to retrieve two components of the symmetric and antisymmetric methylene stretching modes, to lift the degeneracy of the methyl antisymmetric stretching (Figure $3 \mathrm{~A}$ ) and to isolate the methylene scissors mode of TTT conformers, at $1445 \mathrm{~cm}^{-1}$ (Figure 3B). The deconvolution results obtained for the binary mixtures are summarized in Figures S1 and S2 and in Table 1.

As a general trend, the $\mathrm{CH}$ stretching components of $n$ hexane shift to higher wavenumbers with increasing $\mathrm{PFH}$ content, and the intensity ratio between the antisymmetric and symmetric components of the $\mathrm{CH}_{2}$ stretching modes $\left[A_{\text {Total }}\left(\nu_{\text {as }} \mathrm{CH}_{2}\right) / A_{\text {Total }}\left(\nu_{\mathrm{s}} \mathrm{CH}_{2}\right)\right]$ increases, confirming the tendency toward conformers with gauche rotations.

Assuming that the component at $1445 \mathrm{~cm}^{-1}\left(\delta_{\mathrm{sc}} \mathrm{CH}_{2}\right)$ is related exclusively to the all-trans conformer and the one at $1460 \mathrm{~cm}^{-1}\left(\delta_{\mathrm{as}} \mathrm{CH}_{3}\right.$ and $\left.\delta_{\mathrm{sc}} \mathrm{CH}_{2}\right)$ is related exclusively to alltrans and gauche conformers (TTT, TTG and TGT), the evolution of the all-trans conformer proportion with composition may be evaluated by the ratio $\left[A_{1445} /\left(A_{1445}+\right.\right.$ $\left.\left.A_{1460}\right)\right]$. The spectrum of the mixture with $x(\mathrm{PFH})=0.8$ was not analyzed due to its very low intensity in this region. The results (last two rows in Table 1) confirm those obtained for the stretching region: the proportion of all-trans conformers falls from $32 \%$ in pure $n$-hexane to values well below $17 \%$. Moreover, the sharp increase in the $\left[A_{\mathrm{T}}\left(\nu_{\mathrm{as}} \mathrm{CH}_{2}\right) / A_{\mathrm{T}}\left(\nu_{\mathrm{s}} \mathrm{CH}_{2}\right)\right]$ ratio above $x(\mathrm{PFH})=0.6$ is a good indication of a very low
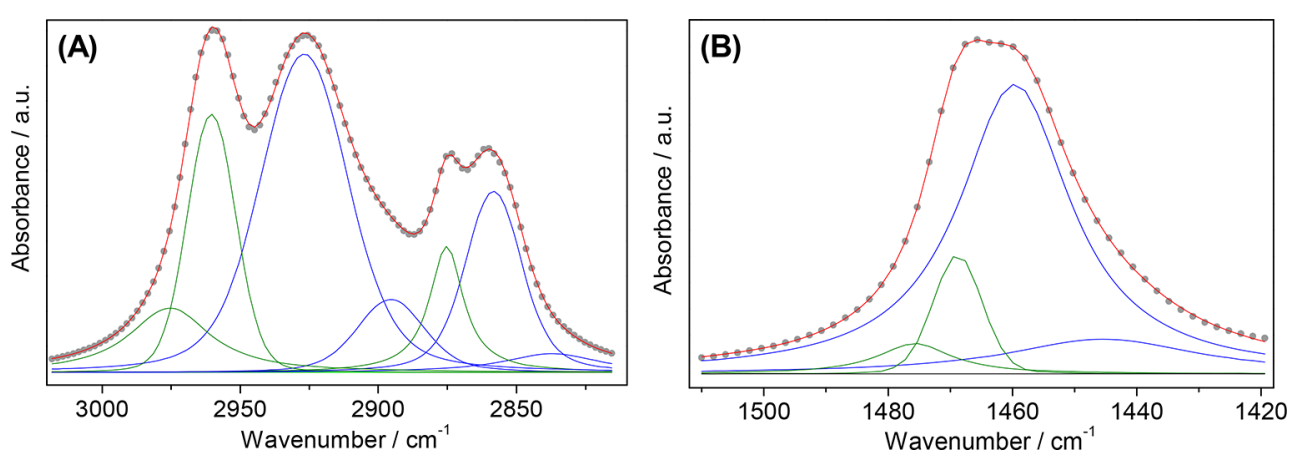

Figure 3. Deconvolution of the infrared spectrum of pure $n$-hexane in a sum of Voigt components for (A) the $\mathrm{CH}$ stretching region and (B) the $\mathrm{CH}$ deformation region. Blue lines, methylene components; green lines, methyl components. 
Table 1. Summary of the Band Deconvolution Results in the $\mathrm{CH}$ Stretching and Deformation Regions for Pure $n$-Hexane and (n-Hexane + Perfluorohexane) Mixtures $^{a}$

\begin{tabular}{|c|c|c|c|c|c|c|c|c|}
\hline \multirow[b]{2}{*}{ assignment $^{34,44}$} & \multicolumn{2}{|c|}{$x(\mathrm{PFH})=0$} & \multicolumn{2}{|c|}{$x(\mathrm{PFH})=0.2$} & \multicolumn{2}{|c|}{$x(\mathrm{PFH})=0.5$} & \multicolumn{2}{|c|}{$x(\mathrm{PFH})=0.8$} \\
\hline & $\tilde{\nu}\left(\mathrm{cm}^{-1}\right)$ & $A(\%)$ & $\tilde{\nu}\left(\mathrm{cm}^{-1}\right)$ & $A(\%)$ & $\tilde{\nu}\left(\mathrm{cm}^{-1}\right)$ & $A(\%)$ & $\tilde{\nu}\left(\mathrm{cm}^{-1}\right)$ & $A(\%)$ \\
\hline \multirow[t]{2}{*}{$\nu_{\mathrm{s}} \mathrm{CH}_{2}$} & 2837 & 2.7 & 2834 & 3.4 & 2836 & 1.6 & 2841 & 3.6 \\
\hline & 2858 & 15.2 & 2858 & 14.5 & 2862 & 14.0 & 2866 & 10.8 \\
\hline$\nu_{\mathrm{s}} \mathrm{CH}_{3}$ & 2875 & 8.4 & 2875 & 8.3 & 2880 & 8.7 & 2883 & 6.4 \\
\hline \multirow[t]{2}{*}{$\nu_{\mathrm{as}} \mathrm{CH}_{2}$} & 2895 & 7.3 & 2893 & 9.3 & 2900 & 6.0 & 2910 & 5.7 \\
\hline & 2927 & 40.4 & 2927 & 39.1 & 2931 & 39.1 & 2937 & 55.2 \\
\hline \multirow[t]{2}{*}{$\nu_{\text {as }} \mathrm{CH}_{3}$} & 2960 & 17.4 & 2961 & 18.3 & 2964 & 17.5 & 2966 & 7.0 \\
\hline & 2975 & 8.7 & 2981 & 7.2 & 2977 & 13.1 & 2974 & 11.3 \\
\hline \multirow[t]{2}{*}{$A_{\mathrm{T}}\left(\nu_{\mathrm{as}} \mathrm{CH}_{2}\right) / A_{\mathrm{T}}\left(\nu_{\mathrm{s}} \mathrm{CH}_{2}\right)$} & \multirow{2}{*}{\multicolumn{2}{|c|}{2.67}} & \multirow{2}{*}{\multicolumn{2}{|c|}{2.71}} & \multicolumn{2}{|c|}{2.89} & \multirow{2}{*}{\multicolumn{2}{|c|}{4.22}} \\
\hline & & & & & 1412 & 11.9 & & \\
\hline$\delta_{\mathrm{sc}} \mathrm{CH}_{2}(\mathrm{TTT})$ & 1445 & 12.4 & 1443 & 9.7 & 1447 & 4.7 & & \\
\hline$\delta_{\mathrm{as}} \mathrm{CH}_{3}(\mathrm{TTT}+\mathrm{TTG})$ and $\delta_{\mathrm{sc}} \mathrm{CH}_{2}(\mathrm{TGT})$ & 1460 & 72.1 & 1459 & 73.1 & 1461 & 56.7 & & \\
\hline$\delta_{\text {as }} \mathrm{CH}_{3}$ & 1469 & 10.2 & 1469 & 14.2 & 1471 & 22.0 & & \\
\hline$\delta_{\mathrm{as}} \mathrm{CH}_{3}$ & 1476 & 5.3 & 1474 & 3.0 & 1474 & 4.7 & & \\
\hline $100 \times\left[A_{1445} /\left(A_{1445}+A_{1460}\right)\right]^{b}$ & 14.6 & & 11.7 & & 7.7 & & & \\
\hline$\% \mathrm{TTT}^{c}$ & & & & & & & & \\
\hline
\end{tabular}

${ }^{a}$ In the deformation region, the band assignment includes the associated rotational isomer. ${ }^{b}$ Ratio between the fitted areas of the indicated components. ${ }^{c}$ Scaled to the $\%$ of TTT referred to in refs 33 and 34 for liquid $n$-hexane.

proportion of the TTT conformer. Therefore, the infrared spectra unambiguously indicate that the addition of $\mathrm{PFH}$ induces coiling on the $n$-hexane chain, shifting the conformational equilibrium toward conformers with more gauche rotations.

Information on $\mathrm{PFH}$ may be retrieved from two spectral ranges: $1375-1025 \mathrm{~cm}^{-1}$, the CF stretching region without a significant contribution from the $n$-hexane spectrum, and 1025-625 $\mathrm{cm}^{-1}$, with strong/medium PFH bands partially overlapped with weak/very weak bands of $n$-hexane. There are several PFH bands that can be correlated with conformers, ${ }^{47}$ in particular, the $\mathrm{CF}_{3}$ deformations at 795 and $712 \mathrm{~cm}^{-1}$ (symmetric and antisymmetric of TTT conformer) and at 816 and $731 \mathrm{~cm}^{-1}$ (symmetric and antisymmetric of TTG conformer). ${ }^{46}$ As previously stated, the PFH conformer referred to as TTT is not a truly all-trans, as the F atoms are not eclipsed. Apparently, mixing with $n$-hexane induced no systematic band shifts or changes in relative intensities (Figure 2C). The analysis was complemented with a band deconvolution in Voigt components (Figure S3 and Table S2), which showed that the relative intensity of the PFH band component at $795 \mathrm{~cm}^{-1}$ is not sensitive to the increasing content in $n$ hexane, suggesting that the population of TTT conformers remains stable. If $n$-hexane perturbs the structure of liquid $\mathrm{PFH}$, then it is not sufficient to change the infrared bands sensitive to rotational conformations. This suggests the possibility of segregation between the two substances, which, given their tendency to phase separation, is not physically unreasonable.

The above conclusions on the mutual conformational influence of the two components according to the infrared analysis are graphically summarized in Figure 4.

In order to obtain further molecular-level support of the hypothesis of segregation, atomistic molecular dynamics (MD) simulations were performed. The OPLS-AA force-field was used, ${ }^{49,50}$ with the dispersive cross-interaction parameters between alkyl hydrogen and perfluoroalkyl fluorine atoms adjusted to capture the properties of (alkane + perfluoroalkane) mixtures: the $\mathrm{H}-\mathrm{F}$ cross-interaction energy was reduced by $20 \%$, and the cross-interaction diameter was increased by $4 \%$ relative to the geometric mean rule. These adjustments were
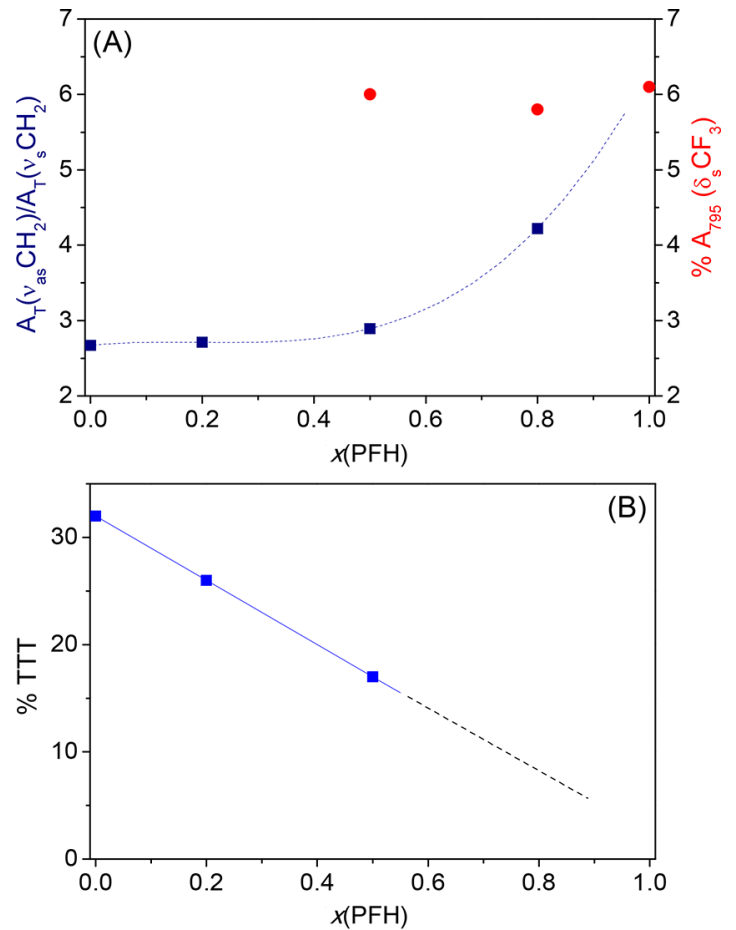

Figure 4. (A) Influence of the second component on the spectra of $n$ hexane (left) and PFH (right): the ratio $A_{\mathrm{T}}\left(\nu_{\mathrm{as}} \mathrm{CH}_{2}\right) / A_{\mathrm{T}}\left(\nu_{\mathrm{s}} \mathrm{CH}_{2}\right)$ is related to the population of $n$-hexane gauche conformers; $A_{795}\left(\delta_{\mathrm{s}} \mathrm{CF}_{3}\right)$ is related to the population of TTT conformers of PFH. (B) Influence of PFH content on the population of $n$-hexane TTT conformers.

obtained in a previous work and reproduce the experimental excess enthalpy and volume. ${ }^{4,51-54} \mathrm{MD}$ runs in the $N p T$ ensemble at $298.15 \mathrm{~K}$ and 1 bar, using the Nosé-Hoover thermostat and barostat, were done using the DL-POLY Classic package $^{55}$ for systems containing 256 molecules in cubic boxes with periodic boundary conditions. The simulations were run for $10 \mathrm{~ns}$, with a time step of $2 \mathrm{fs}$, and the second half of the simulation was used for data collection. Bonds involving hydrogen atoms were treated as rigid using the SHAKE 
algorithm. A 14 A cutoff was used, with standard analytical tail corrections for the dispersion terms and Ewald sum calculations for long-range electrostatics.

Carbon-carbon radial distribution functions (rdf) are shown in Figure 5 for $(n$-hexane + PFH $)$ mixtures. As can be seen,

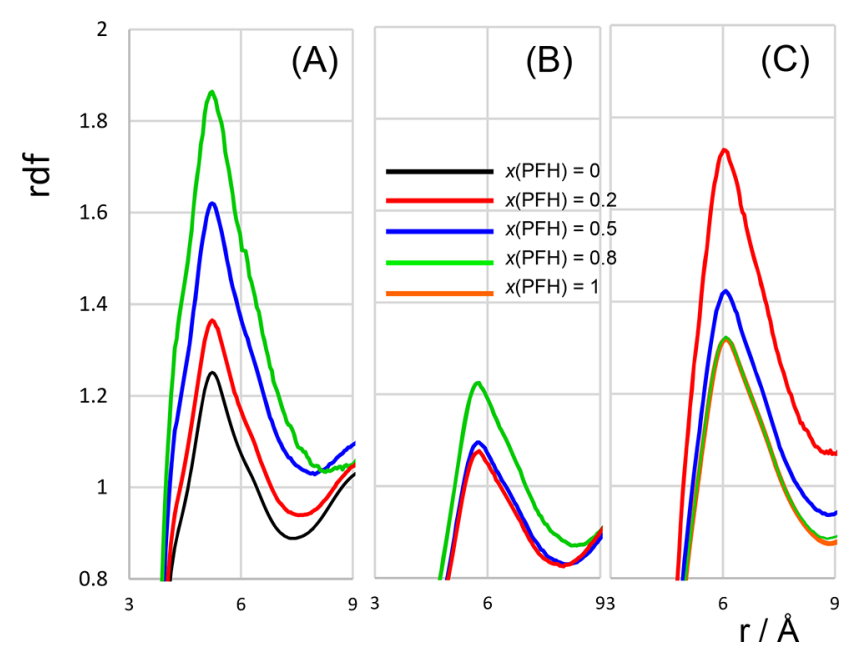

Figure 5. Intermolecular radial distribution functions between (A) $\mathrm{CH}_{n}$ groups, (B) $\mathrm{CH}_{n}-\mathrm{CF}_{n}$ groups, and (C) $\mathrm{CF}_{n}$ groups for (n-hexane $+\mathrm{PFH})$ mixtures at $298 \mathrm{~K}$.

peaks between hydrogenated chains increase significantly as the concentration of PFH increases and are always much larger than that of pure hexane. Similar behavior is found for peaks between fluorinated chains: they increase as the concentration of $n$-hexane increases, although with a weaker dependency on composition. This is a clear sign of segregation as, for both substances, the relative density of neighbors of the same species is larger in the mixtures than in the pure liquids. It is also seen that peaks between hydrogenated and fluorinated groups are much less intense (Figure 5B).

The dihedral distribution and end-to-end distances of the hydrogenated chains were also obtained from the simulation trajectories. However, the simulation results do not show any conformational differences from pure hexane for hexane in perfluorohexane solutions, contrary to what is observed experimentally. We believe that this is an indication that the conformational model used in the simulations is either not adequate or insufficiently accurate to capture the new reported effect.

Extending the present study to other (alkane + perfluoroalkane) mixtures poses significant experimental difficulties due to liquid-liquid immiscibility in the case of mixtures involving longer components or too high a volatility if shorter compounds are used. Nevertheless, we obtained FTIR spectra of the coexisting phases of an $(n$-octane $+\mathrm{PFH})$ mixture. The effect of PFH on the conformational equilibrium of $n$-octane is clearly seen in Figure 6, which shows shifts of the $\mathrm{CH}_{2}$ stretching bands comparable to those observed for ( $n$-hexane $+\mathrm{PFH})$, denoting coiling of the $n$-octane molecules.

The potential coiling of $n$-alkanes in water and aqueous systems is a very important subject and has been addressed and discussed repeatedly in the literature..$^{58,59}$ It should be emphasized that, given the completely different nature of the solvents, the coiling of $n$-alkanes in perfluoroalkanes, now reported, and in water occurs through different mechanisms.

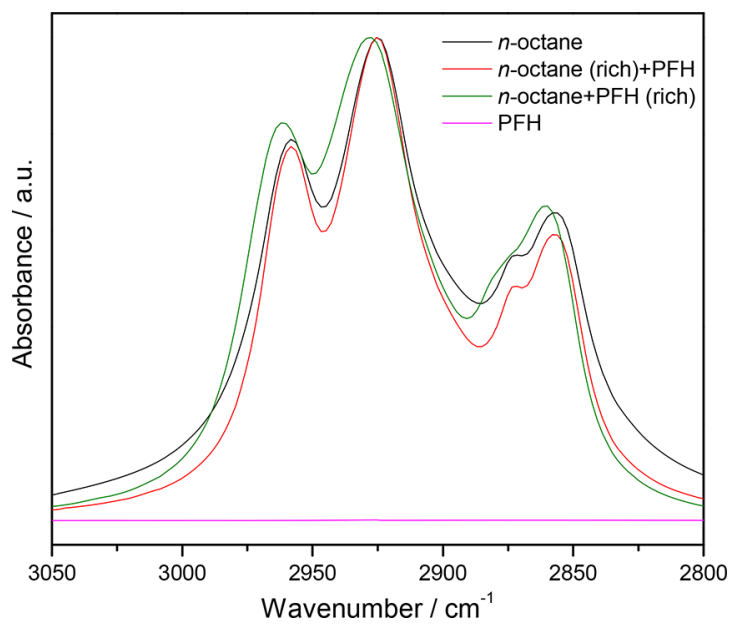

Figure 6. $\mathrm{CH}$ stretching region of the IR spectra of $n$-octane, $\mathrm{PFH}$, and their coexisting liquid phases at $\sim 294 \mathrm{~K}^{56,57}$

In the case of water, coiling, segregation, and ultimately phase separation should result from the maximization of the strong intermolecular forces between water molecules (hydrophobic effect); in the case of the (alkane + perfluoroalkane) mixtures studied in this work, only dispersion forces are present, and as previously discussed, those between alkanes and perfluoroalkanes are unexpectedly weak. In this case, we believe that coiling and segregation possibly result from trying to maximize alkane-alkane and perfluoroalkane-perfluoroalkane dispersive interactions. The fact that perfluoroalkanes do not show experimental evidence of coiling agrees with the known stiffness of fluorinated chains compared to the flexibility of hydrogenated chains.

\section{CONCLUSIONS}

It is demonstrated that $n$-alkanes change their conformational equilibria in the presence of perfluoroalkanes, favoring more globular states, with a higher number of gauche conformations. To the best of our knowledge, this is the first time that such a solvophobic coiling effect was observed in mixtures governed solely by dispersion interactions. Moreover, the origin of the observed effect lies on a subtle balance between weak and even weaker dispersion forces. The implications of the novel solvophobic effect with respect to the structure and properties of fluids involving mixed hydrogenated and fluorinated chains are apparent: coiling of hydrogenated chains will influence the organization of the fluid which in turn will impact the thermodynamic properties and phase equilibria. Additional evidence was found for molecular-level segregation in the $(n-$ hexane + perfluorohexane) mixture above the upper critical solution temperature.

\section{ASSOCIATED CONTENT}

\section{Supporting Information}

The Supporting Information is available free of charge on the ACS Publications website at DOI: 10.1021/acs.langmuir.7b02516.

IR band assignment and deconvolution analysis (PDF)

\section{AUTHOR INFORMATION}

\section{Corresponding Authors}

*E-mail: lilharco@tecnico.ulisboa.pt. Tel: +351 218419220. 
*E-mail: efilipe@tecnico.ulisboa.pt. Tel: +351 218419261.

ORCID

Laura M. Ilharco: 0000-0001-6994-1464

Eduardo J. M. Filipe: 0000-0003-4440-7710

Notes

The authors declare no competing financial interest.

\section{ACKNOWLEDGMENTS}

Fundação para a Ciência e Tecnologia, projects UID/NAN/ $50024 / 2013$ and UID/QUI/0100/2013 and grant SFRH/ $\mathrm{BPD} / 81748 / 2011$.

\section{REFERENCES}

(1) Scott, R. L. The Anomalous Behavior of Fluorocarbon Solutions. J. Phys. Chem. 1958, 62, 136-145.

(2) Morgado, P.; Tomás, R.; Zhao, H.; Dos Ramos, M. C.; Blas, F. J.; McCabe, C.; Filipe, E. J. M. Solution Behavior of Perfluoroalkanes and Perfluoroalkylalkane Surfactants in N-Octane. J. Phys. Chem. C 2007, $111,15962-15968$.

(3) Morgado, P.; Rodrigues, H.; Blas, F. J.; McCabe, C.; Filipe, E. J. M. Perfluoroalkanes and Perfluoroalkylalkane Surfactants in Solution: Partial Molar Volumes in N-Octane and Hetero-SAFT-VR Modelling. Fluid Phase Equilib. 2011, 306, 76-81.

(4) Lepori, L.; Matteoli, E.; Spanedda, A.; Duce, C.; Tiné, M. R. Volume Changes on Mixing Perfluoroalkanes with Alkanes or Ethers at 298.15 K. Fluid Phase Equilib. 2002, 201, 119-134.

(5) Morgado, P.; Black, J.; Lewis, J. B.; Iacovella, C. R.; McCabe, C.; Martins, L. F. G.; Filipe, E. J. M. Viscosity of Liquid Systems Involving Hydrogenated and Fluorinated Substances: Liquid Mixtures of (Hexane + Perfluorohexane). Fluid Phase Equilib. 2013, 358, 161-165.

(6) McLure, I. A.; Edmonds, B.; Lal, M. Extremes in Surface Tension of Fluorocarbon + Hydrocarbon Mixtures. Nature, Phys. Sci. 1973, 241, 71.

(7) Handa, T.; Mukerjee, P. Surface Tensions of Nonideal Mixtures of Fluorocarbons and Hydrocarbons and Their Interfacial Tensions Against Water. J. Phys. Chem. 1981, 85, 3916-3920.

(8) Riess, J. G. Perfluorocarbon-Based Oxygen Delivery. Artif. Cell. Blood Sub. 2006, 34, 567-580.

(9) Courrier, H. M.; Vandamme, T. F.; Krafft, M. P. Reverse Waterin-Fluorocarbon Emulsions and Microemulsions Obtained With a Fluorinated Surfactant. Colloids Surf., A 2004, 244, 141-148.

(10) Gerber, F.; Waton, G.; Krafft, M. P.; Vandamme, T. F. Long Lived Microbubbles for Oxygen Delivery. Artif. Cells Blood Substit. Immobil. Biotechnol. 2007, 35, 119-124.

(11) Rossi, S.; Waton, G.; Krafft, M. P. Small Phospholipid-Coated Gas Bubbles Can Last Longer Than Larger Ones. ChemPhysChem 2008, 9, 1982-1985.

(12) Xiao, Q.; Rubien, J. D.; Wang, Z.; Reed, E. H.; Hammer, D. A.; Sahoo, D.; Heiney, P. A.; Yadavalli, S. S.; Goulian, M.; Wilner, S. E.; Baumgart, T.; Vinogradov, S. A.; Klein, M. L.; Percec, V. Self-Sorting and Coassembly of Fluorinated, Hydrogenated, and Hybrid Janus Dendrimers into Dendrimersomes. J. Am. Chem. Soc. 2016, 138, 12655-12663.

(13) Turberg, M. P.; Brady, J. E. Semifluorinated Hydrocarbons Primitive Surfactant Molecules. J. Am. Chem. Soc. 1988, 110, 77977801.

(14) Binks, B. P.; Fletcher, P. D. I.; Kotsev, S. N.; Thompson, R. L. Adsorption and Aggregation of Semifluorinated Alkanes in Binary and Ternary Mixtures with Hydrocarbon and Fluorocarbon Solvents. Langmuir 1997, 13, 6669-6682.

(15) Mahler, W.; Guillon, D.; Skoulios, A. Smectic Liquid Crystal from (Perfluorodecyl) Decane. Mol. Cryst. Liq. Cryst. 1985, 2, 111119.

(16) Viney, C.; Russell, T. P.; Depero, L. E.; Twieg, R. J. Transitions to Liquid-Crystalline Phases in a Semifluorinated Alkane. Mol. Cryst. Liq. Cryst. 1989, 168, 63-82.
(17) Viney, C.; Twieg, R. J.; Russell, T. P.; Depero, L. E. The Structural Basis of Transitions Between Highly Ordered Smectic Phases in Semifluorinated Alkanes. Liq. Cryst. 1989, 5, 1783-1788.

(18) Morgado, P.; Zhao, H.; Blas, F. J.; McCabe, C.; Rebelo, L. P. N.; Filipe, E. J. M. Liquid Phase Behavior of Perfluoroalkylalkane Surfactants. J. Phys. Chem. B 2007, 111, 2856-2863.

(19) Morgado, P.; Lewis, J. B.; Laginhas, C. M. C.; Martins, L. F. G.; McCabe, C.; Blas, F. J.; Filipe, E. J. M. Systems Involving Hydrogenated and Fluorinated Chains: Volumetric Properties of Perfluoroalkanes and Perfluoroalkylalkane Surfactants. J. Phys. Chem. B 2011, 115, 15013-15023.

(20) Morgado, P.; Laginhas, C. M. C.; Lewis, J. B.; McCabe, C.; Martins, L. F. G.; Filipe, E. J. M. Viscosity of Liquid Perfluoroalkanes and Perfluoroalkylalkane Surfactants. J. Phys. Chem. B 2011, 115, 9130-9139.

(21) Morgado, P. Ph.D. Thesis. Instituto Superior Técnico, Lisboa, 2011.

(22) Rowlinson, J. S. Liquids and Liquid Mixtures, 2nd ed.; Butterworth Scientific: London, 1969.

(23) Dantzler Siebert, E. M.; Knobler, C. M. Interaction Virial Coefficients in Hydrocarbon-Fluorocarbon Mixtures. J. Phys. Chem. 1971, 75, 3863-3870.

(24) Song, W.; Rossky, P. J.; Maroncelli, M. Modeling Alkane + Perfluoroalkane Interactions Using All-Atom Potentials: Failure of the Usual Combining Rules. J. Chem. Phys. 2003, 119, 9145-9162.

(25) Archer, A. L.; Amos, M. D.; Jackson, G.; McLure, I. A. The Theoretical Prediction of the Cricital Points of Alkanes, Perfluoroalkanes, and Their Mixtures Using Bonded Hard-Sphere (BHS) Theory. Int. J. Thermophys. 1996, 17, 201-211.

(26) Dias, A. M. A.; Pàmies, J. C.; Coutinho, J. A. P.; Marrucho, I. M.; Vega, L. F. SAFT Modeling of the Solubility of Gases in Perfluoroalkanes. J. Phys. Chem. B 2004, 108, 1450-1457.

(27) Morgado, P.; McCabe, C.; Filipe, E. J. M. Modelling the Phase Behaviour and Excess Properties of Alkane + Perfluoroalkane Binary Mixtures with the SAFT-VR Approach. Fluid Phase Equilib. 2005, 228-229, 389-393.

(28) Lo Nostro, P. Phase Separation Properties of Fluorocarbons, Hydrocarbons and Their Copolymers. Adv. Colloid Interface Sci. 1995, $56,245-287$.

(29) Zhang, L.; Siepmann, J. I. Pressure Dependence of the VaporLiquid-Liquid Phase Behavior in Ternary Mixtures Consisting of NAlkanes, N-Perfluoroalkanes, and Carbon Dioxide. J. Phys. Chem. B 2005, 109, 2911-2919.

(30) Duarte, P.; Silva, M.; Rodrigues, D.; Morgado, P.; Martins, L. F. G.; Filipe, E. J. M. Liquid Mixtures Involving Hydrogenated and Fluorinated Chains: (P, P, T, X) Surface of (Ethanol $+2,2,2-$ Trifluoroethanol), Experimental and Simulation. J. Phys. Chem. B 2013, 117, 9709-9717.

(31) Sheppard, N.; Szasz, G. J. Spectroscopic Studies of Rotational Isomerism 0.3. The Normal Paraffins in the Liquid and Solid States. J. Chem. Phys. 1949, 17, 86-92.

(32) Snyder, R. G. Vibrational Study of Chain Conformation of Liquid n-Paraffins and Molten Polyethylene. J. Chem. Phys. 1967, 47, 1316.

(33) Almarza, N. G.; Enciso, E.; Bermejo, F. J. Monte-Carlo Simulation of Liquid Normal-Alkanes 0.1. Intramolecular Structure and Thermodynamics. J. Chem. Phys. 1992, 96, 4625-4632.

(34) Huang, Y.; Wang, H. An Investigation of the Conformational Behavior of $\mathrm{n}$-Hexane Adsorbed in Zeolites by FT-Raman Spectroscopy. Langmuir 2003, 19, 9706-9713.

(35) Röthlisberger, U.; Laasonen, K.; Klein, M. L.; Sprik, M. The Torsional Potential of Perfluoro n-Alkanes: A Density Functional Study. J. Chem. Phys. 1996, 104, 3692-3700.

(36) Hannon, M. J.; Boerio, F. J.; Koenig, J. L. Vibrational Analysis of Polytetrafluoroethylene. J. Chem. Phys. 1969, 50, 2829.

(37) Albinsson, B.; Michl, J. The Gauche, Ortho, and Anti Conformers of Perfluoro-n-Butane - Matrix Isolation IR-Spectra. J. Am. Chem. Soc. 1995, 117, 6378-6379. 
(38) Jang, S. S.; Blanco, M.; Goddard, W. A.; Caldwell, G.; Ross, R. B. The Source of Helicity in Perfluorinated N -Alkanes. Macromolecules 2003, 36, 5331-5341.

(39) Cormanich, N. A.; O’Hagen, D.; Bühl, M. Hyperconjugation Is the Source of Helicity in Perfluorinated n-Alkanes. Angew. Chem., Int. Ed. 2017, 56, 7867-7870.

(40) Keefe, C. D.; MacDonald, J. L. Optical Constant, Molar Absorption Coefficient, and Imaginary Molar Polarizability Spectra of Liquid Hexane at $25^{\circ} \mathrm{C}$ Extended to $100 \mathrm{~cm}^{-1}$ and Vibrational Assignment and Absolute Integrated Intensities Between 4000 and $100 \mathrm{~cm}^{-1}$. Vib. Spectrosc. 2007, 44, 121-132.

(41) Wong, P. T. T.; Mantsch, H. H. Pressure Effects on the Infrared Spectrum of 1,2-Dipalmitoyl Phosphatidylcholine Bilayers in Water. J. Chem. Phys. 1985, 83, 3268.

(42) Kellar, J. J.; Cross, W. M.; Yalamanchili, M. R.; Young, C. A.; Miller, J. D. Surface Phase Transitions of Adsorbed Collector Molecules as Revealed by in-situ FT-IR/IRS Spectroscopy. Miner. Metall. Proc. 1993, 10, 75-80.

(43) Snyder, R. G.; Hsu, S. L.; Krimm, S. Vibrational Spectra in the $\mathrm{C}-\mathrm{H}$ Stretching Region and the Structure of the Polymethylene Chain. Spectrochim. Acta, Part A 1978, 34, 395-406.

(44) Kavitha, G.; Narayana, C. Raman Spectroscopic Investigations of Pressure-Induced Phase Transitions in $n$-Hexane. J. Phys. Chem. B 2007, 111, 14130-14135.

(45) Morgado, P.; Garcia, A. R.; Ilharco, L. M.; Marcos, J.; Anastácio, M.; Martins, L. F. G.; Filipe, E. J. M. Liquid Mixtures Involving Hydrogenated and Fluorinated Alcohols: Thermodynamics, Spectroscopy, and Simulation. J. Phys. Chem. B 2016, 120, 10091-10105.

(46) Zoellner, R. W.; Latham, C. D.; Goss, J. P.; Golden, W. G.; Jones, R.; Briddon, P. R. The Structures and Properties of Tetrafluoromethane, Hexafluoroethane, and Octafluoropropane Using the AIMPRO Density Functional Program. J. Fluorine Chem. 2003, 121, 193-199.

(47) Pirozhnaya, L. N.; Zubkova, O. B. Calculation of Vibrational Spectra and Rotational Isomerism of Perfluoroparaffins. $n-\mathrm{C}_{4} \mathrm{~F}_{10}$ and n- $\mathrm{C}_{6} \mathrm{~F}_{14}$ Molecules. J. Struct. Chem. 1985, 26, 908-912.

(48) Axford, D. W. E.; Rank, D. H. Spectroscopic Studies of Rotational Isomerism. V. The Infra-Red Absorption Spectra of Six Solid Hydrocarbons in the Region $1600-650 \mathrm{~cm}^{-1}$. J. Chem. Phys. 1950, 18, 51.

(49) Jorgensen, W. L.; Maxwell, D. S.; Tirado-Rives, J. Development and Testing of the OPLS All-Atom Force Field on Conformational Energetics and Properties of Organic Liquids. J. Am. Chem. Soc. 1996, $118,11225-11236$.

(50) Watkins, E. K.; Jorgensen, W. L. Perfluoroalkanes: Conformational Analysis and Liquid-State Properties from $\mathrm{Ab}$ Initio and Monte Carlo Calculations. J. Phys. Chem. A 2001, 105, 4118-4125.

(51) Williamson, A. G.; Scott, R. L. Heats of Mixing of NonElectrolyte Solutions. II. Perfluoro-N-Heptane + Isooctane and Perfluoro-N-Hexane + N-Hexane. J. Phys. Chem. 1961, 65, 275-279.

(52) Matteoli, E.; Lepori, L. Determination of the Excess Enthalpy of Binary Mixtures from the Measurements of the Heat of Solution of the Components: Application to the Perfluorohexane + Hexane Mixture. Fluid Phase Equilib. 2000, 174, 115-131.

(53) Bedford, R. G.; Dunlap, R. D. Solubilities and Volume Changes Attending Mixing for the System: Perfluoro-N-Hexane-N-Hexane. J. Am. Chem. Soc. 1958, 80, 282-285.

(54) Morgado, P.; Martins, L. F. G.; Filipe, E. J. M. Probing the Structure of Liquids with ${ }^{129}$ Xe NMR Spectroscopy: Perfluoroalkanes and Alkane + Perfluoroalkane Mixtures. Submitted to J. Phys. Chem. B. (55) Smith, W.; Forester, T. R.; Todorov, I. T. The DL_POLY Classic User Manual, version 1.9; Daresbury Laboratory: United Kingdom, 2012.

(56) Duce, C.; Tiné, M. R.; Lepori, L.; Matteoli, E. VLE and LLE of Perfluoroalkane + Alkane Mixtures. Fluid Phase Equilib. 2002, 199, 197-212.

(57) Lo Nostro, P.; Scalise, L.; Baglioni, P. Phase Separation in Binary Mixtures Containing Linear Perfluoroalkanes. J. Chem. Eng. Data 2005, 50, 1148-1152.
(58) Sun, L.; Siepmann, J. I.; Schure, M. R. Conformation and Solvation Structure for an Isolated n-Octadecane Chain in Water, Methanol, and Their Mixtures. J. Phys. Chem. B 2006, 110, 1051910525.

(59) Ferguson, A. L.; Debenedetti, P. G.; Panagiotopoulos, A. Z. Solubility and Molecular Conformations of n-Alkane Chains in Water. J. Phys. Chem. B 2009, 113, 6405-6414. 